\title{
LOS VALORES CÍVICOS EN LOS TEXTOS DEL GRADO DE EDUCACIÓN PRIMARIA
}

\author{
Eva Ortiz Cermeño \\ Tomás Izquierdo Rus \\ Pedro Miralles Martínez \\ Universidad de Murcia
}

\begin{abstract}
RESUMEN: Se presenta un estudio de los manuales docentes universitarios sobre los valores cívicos en el Grado de Educación Primaria en las universidades españolas. Los datos obtenidos se analizan con el programa ATLAS.ti versión 6.2. Los resultados muestran cómo las teorías analizadas inciden en los valores de la libertad y la disciplina principalmente, seguidos del respeto, la igualdad relacionada con la justicia, la responsabilidad y la participación en estrecha relación con la participación ciudadana. Se concluye que los valores que se trabajan en esta asignatura son esenciales en la formación inicial de los maestros y se sugieren nuevas líneas de investigación en esta temática.
\end{abstract}

PALABRAS CLAVE: Valores cívicos, educación primaria, formación de maestros, ATLAS.ti.

\section{CIVIC VALUES IN THE TEXTS OF THE PRIMARY EDUCATION}

ABSTRACT: Documentary study university textbooks on civic values in the Primary Education. Technique has been used as discourse analysis with ATLAS.ti version 6.2. The results show how the theories discussed affect the values of freedom and discipline primarily, followed by respect, equality related to justice, accountability and participation closely with citizen participation. We conclude that the values that work in this subjet are essential in the initial training of teachers.

KEYWORDS: Civic values, primary education, teacher training, ATLAS.ti.

Recibido: 10/07/2014

Aceptado: 22/09/2014 


\section{INTRODUCCIÓN}

La educación en valores constituye uno de los principales retos educativos de la actualidad. Por ello, en los últimos años todas las reformas educativas de nuestro entorno cultural han contemplado el concepto de temas o ejes transversales como elementos curriculares que se introducen a lo largo de todo el proceso educativo para desarrollar determinados valores. Comentan Paredes y Ribera (2006) que es importante coordinar y tratar de manera conjunta los valores desarrollados a través del currículo para que no queden descontextualizados, porque los valores son un marco de referencia personal que orienta a las personas en sus acciones, opiniones y decisiones. Los valores se construyen a partir de la relación de cada individuo con su entorno cultural y familiar, sus experiencias de vida, su propia capacidad de análisis, etc. La educación en valores consiste en preparar para aprender a convivir y a profundizar en valores éticos y democráticos esenciales en la sociedad actual. Los valores no se enseñan, se aprenden.

La selección de unos valores, y no otros, es siempre arriesgada, y más todavía cuando incide en algo tan trascendental como la educación. Escoger es renunciar, y renunciar es siempre comprometido. De todas maneras, como afirman Pujol y Luz (2003), los valores humanos se entrelazan entre ellos y resulta difícil distinguir dónde termina uno y dónde empieza otro. Dicho de otro modo, no es factible discernir si estamos educando en el diálogo o en la paz o en la justicia, porque cabría preguntarse: ¿Es posible una paz sin diálogo? ¿Podría existir la paz al margen de la justicia? ¿La urbanidad no es un aspecto del respeto? ¿Es concebible la amistad sin la sinceridad? ¿Y una responsabilidad carente de prudencia? Por ello, no podemos ser más tolerantes sin ser a la vez más generosos, más comprensivos, más dialogantes, más respetuosos, etc. Por ello, "aceptar los valores vigentes (conservarlos y aumentarlos), o bien cambiar la situación dada por antivaliosa es siempre el problema y el quehacer de toda educación" (Gervilla, 1992: 432).

Los valores son cualidades que las personas y los pueblos, a lo largo de la historia o en estos tiempos, descubrimos o ponemos en los acontecimientos, las situaciones y personas para hacer nuestro mundo más habitable, más humano. La reflexión ética de nuestro tiempo ha puesto de manifiesto que determinados valores como la dignidad de la persona, la libertad, la justicia, la igualdad, la solidaridad, el pluralismo político o la democracia, el civismo, la tolerancia, la participación, la paz y la responsabilidad son los anclajes de la ciudadanía moderna.

De acuerdo con Román y Cano (2008), el siglo XIX fue testigo de la gestación, nacimiento e institucionalización en Europa y -en España- de las Escuelas Normales como centros encargados de la formación de maestros de instrucción elemental, responsables de la difusión de la generalización de la educación entre las clases populares. Se plantea la educación en valores como "la transmisión de contenidos informativos, procedimentales y de actitudes, valores y normas que supongan un aprendizaje que permita realizar un análisis crítico de nuestras culturas y de las de los demás y un avance comunicacional en la construcción de una cultura compartida" (Buxarrais y Martínez, 2009: 10).

En el concepto de ciudadanía confluyen dos aspectos de la vida humana: el componente "racional", el de una sociedad que debe ser justa para que sus miembros 
perciban su legitimidad, y el componente "sentimental", representado por esos lazos de pertenencia, que no hemos elegido sino que forman ya parte de nuestra identidad por el mero hecho de nacer en el seno de un determinado país. Ante los retos a los que se enfrenta cualquier comunidad, es entonces posible apelar a la razón y al sentimiento de sus miembros, ya que estos se consideran ciudadanos de esa comunidad, y ésta los considera como cosa suya. Parece, pues, que la racionalidad de la justicia y el sentimiento de pertenencia a una comunidad concreta han de ir a la par, si deseamos asegurar ciudadanos plenos y, a la vez, una democracia sostenible. Esta es la razón por la cual desde la década de los noventa se pone de actualidad el concepto de ciudadanía. Este concepto presenta hoy múltiples facetas (ciudadanía política, social, económica, civil, intercultural y cosmopolita).

Educar para la ciudadanía es "una necesidad actual (y no tanto un oportunismo político), que cobra sentido desde la concepción del aprendizaje como proceso que se desarrolla a lo largo de la vida y que nos permite hacer frente a la realidad de cada momento y contexto" (Puig, Domene y Morales, 2010: 87). Como argumenta Prats (2011), la formación para la ciudadanía en la Universidad es una clara prioridad, según la "Declaración Mundial sobre la Educación Superior en el siglo XXI" de la UNESCO. En su artículo primero, el sistema universitario debe "contribuir a proteger y consolidar los valores de la sociedad, velando por inculcar en los jóvenes los principios en que reposa la ciudadanía democrática". En suma, promover "la formación en valores ciudadanos de los miembros de la comunidad universitaria". Asimismo, este autor comenta que se puede afirmar que la formación ciudadana en valores democráticos no es prioritaria ni rige las preocupaciones y las decisiones cotidianas de nuestras universidades, aunque existe una sensibilidad que impregna, de manera transversal, el ambiente intelectual de la comunidad universitaria. Las instituciones educativas se convierten, de esta manera, en una herramienta para la construcción, el debate y la consolidación del ejercicio de la ciudadanía (Torres, 2013).

Adentrarse en la educación cívica conlleva que hay unos valores mínimos esenciales para una vida digna (paz, libertad, igualdad, justicia y solidaridad) y unos principios de una vida en común (responsabilidad, tolerancia, diálogo, honestidad, civismo, etc.), de los que se derivan normas, hábitos y actitudes (Pérez Tapias, 1996). Además de estos valores generales, se han unido todos aquellos que, en un nuevo humanismo, nos preocupan en nuestro presente y futuro inmediato (medio, salud y convivencia), en especial el derecho a vivir juntos en un planeta sano, con una responsabilidad compartida por el futuro, que recogen los temas transversales (Bolívar, 2000). Dirigirse hacia una educación cívica activa en sociedades pluralistas y democráticas supone conocer sus derechos y deberes como ciudadanos y saber ejercerlos, responsabilizarse de sus acciones, ponerse en la piel del "otro" para desarrollar la actitud de acogida a ese "otro" y ser capaz de construir un modelo de vida con algún criterio que permita la construcción de comunidad de un mundo compartido, justo y solidario (Buxarrais y Martínez, 2009).

La libertad puede ser entendida como ser y sentirse libre con el estilo de vida que llevemos y que con nuestra forma de ser y de vivir seamos capaces de afrontar y resolver nuestros problemas del día a día. Asimismo, la libertad es una tarea o conquista personal y social que implica responsabilidad con nosotros mismos y con los otros. De ahí que haya que fomentar en el educando el pensamiento crítico, es decir, 
que debatan, opinen, analicen situaciones, etc. Por tanto, los valores que hay que trabajar para la libertad son entre otros: el reconocimiento del otro, el trato justo, el reconocimiento de la identidad cultural y ser responsable del otro. La libertad es un valor fundamental en la Declaración Universal de los Derechos Humanos, aprobada y proclamada por la Asamblea General de las Naciones Unidas en la histórica sesión del 10 de diciembre de 1948, en París. Esta declaración la repite treinta veces, ya sea como nombre (libertad), como adjetivo (libre), como adverbio (libremente) o como verbo (liberar). Por la libertad se ha luchado, se ha muerto, se ha sufrido, se ha soñado, se ha escrito; ha habido guerras, revoluciones, amotinamientos, Ilantos, risas, héroes, mártires; han surgido teorías filosóficas, tendencias pedagógicas, movimientos sociales, dictaduras y anarquías. "Nos hallamos ante el valor que nos puede traer los mayores disgustos. $\mathrm{Y}$, a pesar de todo, debemos educar en la libertad" (Pujol y Luz, 2003: 155).

Otro valor tratado ha sido el de justicia. La aportación de Aristóteles al concepto de justicia ha marcado buena parte del pensamiento ético occidental. En sentido amplio, concibe la justicia como el medio preferente para alcanzar la felicidad o el bien. La justicia universal consiste básicamente en obedecer la ley, aquella que se halla en el marco del Estado; según este concepto "la justicia es una cuestión de lo justo, lo igual" (Maclntyre, 1994: 127). Se atribuye la cualidad de lo justo, como afirmaba Aristóteles (1984), al que obedece las leyes y al que observa con los demás las reglas de la igualdad. Así lo justo será lo conforme a la ley y a la igualdad, y lo injusto será lo ilegal y lo desigual. De este modo, el núcleo central de la justicia lo constituyen la igualdad y la legalidad. La justicia tiene el carácter de valor mínimo y necesario, ya que es una condición necesaria para que nuestras relaciones con los demás sean correctas. Si no somos justos, no podemos acceder a los demás valores. De todas formas, en la mayoría de las ocasiones, que una cosa sea justa o que no lo sea no depende de nosotros; "la justicia tiene una medida exacta para ser cumplida. Como argumenta Osorio (2010), existen muchas formas de justicia y muchas cosas de las que decimos que son justas e injustas.

El respeto activo es otro de los valores, pues no es difícil encontrar en la sociedad en la que convivimos personas que tienen actitudes y comportamientos distintos al nuestro y que a veces mostramos actitudes y comportamientos de respeto a las ideas y creencias del otro pero otras veces no. Nos encontramos cada vez más en nuestro entorno, xenofobia, rechazo por las diferencias culturales, o por la forma de ser, pensar o actuar; por ello, hay que trabajar en los centros educativos la tolerancia para formar buenos ciudadanos y formarse en valores que traten este tema junto con la diversidad, la convivencia, el respeto al diferente, etc. Así pues, ser tolerante significa respetar y defender el derecho a la libre expresión de las opiniones y formas de ser de los demás, y respetuosos con los valores humanos de todos. De acuerdo con Pujol y Luz (2003: 105) tolerar es entendido como "el significado de reconocer el pluralismo; respetar la diversidad; compartir con los demás las diferencias como algo positivo, beneficioso, enriquecedor, etc.". Sin embargo, la sola tolerancia tiene el inconveniente de poder convertirse fácilmente en indiferencia y, entonces, más que interés porque el otro pueda vivir según sus convicciones y sus criterios es sencillamente desinterés, dejar que el otro se las componga como pueda. Por eso la tolerancia, así entendida, es todavía un valor bastante inferior al verdaderamente positivo, 
que es más que tolerancia, respeto activo. Como comentan Cortina, Escámez y Pérez (1996), consiste en el interés por comprender a otros y por ayudarles a llevar adelante sus planes de vida. El respeto activo es un valor menos estimado hoy que la tolerancia pasiva. Dejar que otros hagan, con tal de que a mí también me dejen en paz, es casi una consigna en las sociedades con democracia liberal. Incluso la tolerancia pasiva acaba desapareciendo si no tiene su base en un aprecio positivo del otro, porque a la larga, en cuanto el otro incomoda, podemos pasar a ser intolerantes, ya que no le apreciamos realmente. Por eso urge en la educación fomentar el respeto activo por los otros, que normalmente desemboca en solidaridad.

El civismo tiende a evitar las incomodidades entre las personas que se relacionan entre sí. Se trata de aplicar unas pautas mínimas de comportamiento que permitan convivir en paz y libertad, respetando a las personas que nos rodean y al entorno en general. La palabra civismo tiene la misma etimología que la palabra civilización, provienen del latín cives que significa ciudadano. Así, saber ser un buen ciudadano es saber mantener una actitud civilizada. El civismo intenta hacer el trato humano lo más agradable posible a través de un comportamiento que respeta unas normas y unas costumbres sociales. Como argumentan Paredes y Ribera (2006), preocuparse por la tolerancia y la convivencia, reclamar respeto para todo tipo de personas, intentar no herir susceptibilidades y preocupase por limar la hostilidad en las relaciones entre las personas no debe dejar de lado el preocuparse por conseguir una actitud más cívica en la sociedad actual. Hablar de civismo es hablar de ciudadanía, de un valor que significa mucho más que urbanidad. El civismo diferencia a la persona que se interesa por su función de ciudadano comprometido y solidario con un espacio común de convivencia compartido con otros ciudadanos. La sociedad se construye gracias a ciudadanos conocedores de sus derechos pero también de sus deberes y que saben armonizar su libertad personal con la responsabilidad solidaria. Los buenos modales y las normas de urbanidad son importantes, pero el civismo es más que eso. El civismo no es un formalismo que hay que cumplir sino que es un sentimiento ético que permite el desarrollo de una cultura de respeto entre personas que comparten un mismo entorno social. "Mejorar el civismo es responsabilidad de todas y cada una de las personas que componemos la sociedad" (Paredes y Ribera, 2006: 148).

En último lugar, en relación a la participación y la responsabilidad en la escuela, ésta debe estar organizada democráticamente de modo que permita la participación, toma de decisiones, compromiso, responsabilidad y puesta en acción de los valores democráticos. "El pensamiento crítico es una forma de pensar de manera responsable relacionada con la capacidad de emitir buenos juicios. Es una forma de pensar por parte de quien está genuinamente interesado en obtener conocimiento y buscar la verdad y no simplemente salir victorioso cuando se está argumentando" (Martín y Barrientos, 2009: 20). Educar a la ciudadanía, incluidas las propias familias, supone primar la participación y la responsabilidad en todos los ámbitos escolares, como una comunidad que comparte por igual un conjunto de derechos democráticos de participación y comunicación. Por ello, formar ciudadanos significa no sólo enseñar un conjunto de valores propios de una comunidad democrática, sino estructurar el centro y la vida en el aula con procesos de diálogo, de debate, y toma de decisiones en los que la participación activa, y la resolución de los proble- 
mas de la vida en común contribuyen a crear los correspondientes hábitos y virtudes cívicas (Bolívar, 2007). Toda una larga generación de estudios e investigaciones han subrayado que la educación cívica no puede consistir sólo en contenidos para aprender una materia, es decir, en un aprendizaje conceptual, sino en un conjunto de prácticas pedagógicas y educativas que comprenden, al menos, tres componentes: conocimientos, habilidades y actitudes y valores. Como tales, exigen procesos de vivencia en el centro escolar y en la comunidad, que además precisan un cierto grado de consistencia entre ellos. De igual modo, "la ciudadanía ha de construirse localmente, ampliando espacios de participación y lugares donde trabajar conjuntamente" (Bolívar, 2007: 27).

Las investigaciones realizadas en torno a la manualística escolar, en un intento de acercarse a las aulas, están apostando por una historia de las prácticas educativas, utilizando para ello los manuales como fuentes documentales de incalculable valor para nuestra historia escolar. Como afirma Armas (2004), uno de los temas principales de investigación son los manuales escolares que constituyen una fuente de primer orden para indagar la producción y distribución del conocimiento escolar así como su evolución a lo largo del tiempo. Diversas instituciones internacionales, como el Institut National de la Recherche Pédagogique en Francia o el alemán Instituto Eckert han desarrollado proyectos de catalogación y estudio de los manuales escolares; una línea de trabajo que también ha encontrado su reflejo en España es el proyecto Manes (Villalaín, 1997; Escolano, 1997-1998). Las técnicas y los instrumentos de este tipo de estudios muestran análisis de documentos de distintos campos científicos a partir de estudios bibliográficos. Por ejemplo, Valls (2001) comenta que existe un consenso cada vez más amplio entre la comunidad científica dedicada al análisis, a la revisión y, en suma, a la mejora de los textos escolares. Este tipo de estudios ya no pueden quedar limitados a los contenidos de los propios textos escolares, sino que tales investigaciones deben de ampliarse necesariamente a su uso en el contexto de las aulas.

\section{Metodología}

El estudio se enmarca en una propuesta de investigación cualitativa de tipo descriptivo e inductivo, ya que busca conocer los fenómenos desde la perspectiva de los propios individuos (Mella, 2003). La fortaleza de la investigación cualitativa está en su dinamismo y flexibilidad para comprender los fenómenos estudiados (Cáceres, 2003), por tanto se encuadra en un paradigma humanístico-interpretativo o constructivista. En concreto, nos hemos centrado en la semiótica, que se ha desarrollado sobre todo como una metodología para la interpretación de textos-discursos.

\subsection{Objetivo}

Teniendo presente el objetivo de esta investigación se centran en realizar un análisis textual a partir de la revisión teórica sobre el tema objeto de estudio; analizar los valores cívicos de la asignatura Teorías e Instituciones Contemporáneas de Educación y establecer un sistema de categorías para la interpretación y la evolución de los valores cívicos. 


\subsection{Informantes clave}

Los informantes clave de esta investigación son los manuales universitarios de Teorías e Instituciones Contemporáneas de Educación, que se han seleccionado, por un lado, teniendo en cuenta los planes de estudio vigentes y, por otro, atendiendo a los autores del ámbito de la pedagogía más prestigiosos e influyentes en su especialidad y en la asignatura en la que se enmarca este estudio, materia que es clave en la formación inicial de los maestros. Los manuales que han sido objeto de estudio, ordenados por fechas, se relacionan en la Tabla 1.

Tabla 1. Manuales universitarios de la asignatura Teorías e Instituciones Contemporáneas de Educación

Marín, R. (1988). Principios de la educación contemporánea. Madrid: RIALP.

Colom, A. J., Lluís, J., Domínguez, E. y Sarramona, J. (1997). Teorías e instituciones contemporáneas de educación. Barcelona: Ariel.

lyanga, A. (1998). La Educación Contemporánea. Teorías e Instituciones. Valencia: Nau Llibres.

Carreño, M. (Ed.), Colmenar, C., Egido, I. y Sanz, F. (2000). Teorías e Instituciones contemporáneas de educación. Madrid: Síntesis Educación.

García, A. (2001). Teorías e Instituciones de la educación. Una aproximación axiológica. Sevilla: Padilla Libros Editores y Libreros.

Carreño, M. (Ed.), Colmenar, C., Egido, I. y Sanz, F. (2002). Teorías e Instituciones contemporáneas de educación. Madrid: Síntesis Educación.

Colom, A. J., Lluís, J., Domínguez, E. y Sarramona, J. (2002). Teorías e instituciones contemporáneas de educación. Barcelona: Ariel.

Vicente, U. (2002). La educación: Teorías e Instituciones contemporáneas. Almería: Universidad de Almería.

Negrín, O. y Vergara, J. (2003). Teorías e Instituciones contemporáneas de educación. Madrid: Centro de Estudios Ramón Areces.

Del Pozo, M. aㅗ., Álvarez, J. L., Luengo, J. y Otero, E. (2004). Teorías e Instituciones contemporáneas de educación. Madrid: Biblioteca Nueva.

Colom, A. J., Lluís, J., Domínguez, E. y Sarramona, J. (2005). Teorías e instituciones contemporáneas de educación. 4. ed. Barcelona: Ariel.

Negrín, O. y Vergara, J. (2006). Teorías e Instituciones contemporáneas de educación. Madrid: Centro de Estudios Ramón Areces.

Colom, A. J., Lluís, J., Domínguez, E. y Sarramona, J. (2008). Teorías e instituciones contemporáneas de educación. Barcelona: Ariel.

Carreño, M. (Ed.), Colmenar, C., Egido, I. y Sanz, F. (2008). Teorías e Instituciones contemporáneas de educación. Madrid: Síntesis Educación.

Del Pozo, M. ํ. M., Álvarez, J. L., Luengo, J. y Otero, E. (2009). Teorías e Instituciones contemporáneas de educación. 2. ed. Madrid: Biblioteca Nueva.

La asignatura Teorías e instituciones contemporáneas de educación se ha impartido en la formación inicial del profesorado de Primaria y se imparte actualmente en las titulaciones de Grado de Educación Primaria en las universidades españolas. 


\subsection{Análisis de datos}

En función del objetivo de este estudio se seleccionaron los manuales de la asignatura Teorías e Instituciones Contemporáneas de Educación y se estableció un sistema de categorías para el análisis textual de la interpretación y evolución de los valores cívicos de las teorías educativas utilizando el método inductivo mediante el programa informático ATLAS.ti 6.2. Este programa permitirá la relación de tres actividades fundamentales: reducción de datos, disposición y transformación y obtención y verificación de conclusiones. Cada una de estas tareas tiene algunas operaciones particulares que están en constante interacción a lo largo de todo el procedimiento analítico, tal y como lo llevan a cabo, por ejemplo, Rodríguez, Gil y García Giménez (1996) y Pérez (1994). La búsqueda en los textos de las citas, usando categorías, es una de las herramientas más poderosas del ATLAS.ti y es muy útil cuando se tiene mucho material informativo. Asimismo, este programa resulta eficaz para la elaboración y representación de redes semánticas de contenido en el análisis textual. En cada teoría o corriente estudiada se inciden en los valores comentados como queda recogido en la tabla 2.

La técnica de codificación utilizada han sido los diagramas de redes como sistema de representación y proceso de análisis del discurso, porque permiten sintetizar información y descubrir relaciones significativas entre conceptos, facilitando su tratamiento y presentación. Por tanto, se decidió utilizarlos en el análisis del discurso en los libros de texto analizados.

Tabla 2. Teorías y corrientes educativas. Valores cívicos

\begin{tabular}{|c|c|}
\hline Autores & Teorías y corrientes educativas: valores \\
\hline $\begin{array}{l}\text { Bernabeu }(1997,2000,2008) \\
\text { Carreño }(2000,2002,2008) \\
\text { Del Pozo }(2004,2009)\end{array}$ & $\begin{array}{l}\text { Teorías marxistas: educación, formación. } \\
\text { disciplina, formación del educando. } \\
\text { responsabilidad cívica, actitudes. }\end{array}$ \\
\hline $\begin{array}{l}\text { Otero }(2004,2009) \\
\text { Negrín y Vergara }(2003,2006)\end{array}$ & $\begin{array}{l}\text { Teorías contemporáneas: disciplina. } \\
\text { libertad. }\end{array}$ \\
\hline Colom $(1997,2002,2008)$ & Teorías de la posmodernidad: Nietzche no cree en valores. \\
\hline $\begin{array}{l}\text { Bernabeu, Colom }(1997,2002,2008) \text { y } \\
\text { Del Pozo }(2004,2009)\end{array}$ & Paradigma crítico hermenéutico: diálogo, profesor/alumno. \\
\hline $\begin{array}{l}\text { Domínguez }(1997,2002,2008) \text { y } \\
\text { Negrín y Vergara }(2003,2006)\end{array}$ & $\begin{array}{l}\text { Teorías y métodos de la Escuela Nueva: respeto, autonomía, } \\
\text { libertad. }\end{array}$ \\
\hline $\begin{array}{l}\text { Marín (1988) } \\
\text { Carreño }(2000,2002,2008) \text { y Negrín y } \\
\text { Vergara }(2003,2006) \\
\text { Del Pozo }(2004)\end{array}$ & $\begin{array}{l}\text { El movimiento de la Escuela Nueva: disciplina, conciencia moral } \\
\text { y juicios de valor. Autonomía, y experiencia personal. } \\
\text { El movimiento de la Escuela Nueva: libertad, disciplina, } \\
\text { cooperación. } \\
\text { El movimiento de la Escuela Nueva: democracia (participación } \\
\text { activa, pensamiento colectivo de carácter crítico, ciudadanía, } \\
\text { formación del ciudadano. }\end{array}$ \\
\hline $\begin{array}{l}\text { Bernabeu y Colom }(1997,2002,2008) \text {, } \\
\text { Negrín y Vergara }(2003,2006) \text { y } \\
\text { Del Pozo }(2004,2009)\end{array}$ & Teorías antiautoritarias: libertad, responsabilidad, confianza. \\
\hline Negrín y Vergara $(2003,2006)$ & $\begin{array}{l}\text { La escuela moderna y la pedagogía institucional: responsabilidad, } \\
\text { libertad. }\end{array}$ \\
\hline
\end{tabular}




\begin{tabular}{|l|l|}
\hline Negrín y Vergara $(2003,2006)$ & $\begin{array}{l}\text { Teorías de la desescolarización: libertad, formación, autonomía, } \\
\text { respeto, confianza. }\end{array}$ \\
\hline Bernabeu $(1997,2000,2002)$ y & $\begin{array}{l}\text { Teorías personalistas: diálogo, relación de ayuda, libertad, } \\
\text { Negrín y Vergara }(2003,2006)\end{array}$ \\
$\begin{array}{l}\text { Del Pozo }(2004,2009), \text { Negrín y } \\
\text { Vergara }(2003,2006) \text { y }\end{array}$ & $\begin{array}{l}\text { Teorías personalistas: democracia (justicia, igualdad, diálogo, } \\
\text { pensamiento crítico, saber escuchar a los otros, tolerancia). }\end{array}$ \\
\hline
\end{tabular}

Como se observa en la tabla 2, la evolución de los valores según los autores analizados en los manuales se puede sintetizar del siguiente modo:

- Teorías marxistas: Sucholoski (cit. Bernabeu en Colom, 1997) hace referencia a los valores focalizados en el desarrollo social de la persona. Makarenko (cit. Bernabeu en Colom, 1997, Carreño, 2000 y Negrín y Vergara, 2003), destaca valores morales, centrados en la comunidad, la sociedad. Marx y Engels (cit. Del Pozo, 2004) relacionan los valores con las creencias. Gramsci (cit. Bernabeu en Colom, 1997 y Carreño, 2000) desarrolla valores centrados en la autodisciplina y la autonomía moral para poder conseguir una formación académica y personal. Los marxistas enfatizan los valores soviéticos, considerados por el Stalinismo con una considerable carga ideológica.

En estas teorías Suchodolski (cit. Bernabeu en Colom, 1997) se refiere a la formación y al desarrollo social de la persona participando colectivamente. Del Pozo (2004) trabaja lo cívico desde la responsabilidad y la disciplina. Makarenko (cit. Del Pozo, 2004) resalta las actitudes, los valores ideológicos de la escuela, aludiendo a la disciplina fundamentalmente.

Movimiento de la Escuela Nueva: no alude a los valores de forma general sino que hace un tratamiento de forma específica sobre valores concretos.: Marín (1988) hace mención a Luzuriaga y García Benítez (2001) a Planchard para comentar que ambos autores destacan la formación de la persona desde el civismo y la cooperación, con un sentido comunitario y colectivo entre todos. Lietz (cit. Negrín y Vergara (2003) junto con sus colaboradores Wyneken y Geheeb se centran en la coeducación incluyendo en sus programas que los alumnos adquieran y desarrollen caracteres religiosos-cívicos. Comparten estas ideas Rousseau, Pestalozzi, Tolstoi, Key, Froebel y Herbart. Que el ciudadano adquiera preparación realizando juicios de valor en relación a la nación y a la sociedad manteniendo en todo momento una neutralidad confesional.

- Teorías educativas en los umbrales de la contemporaneidad: Iyanga (1998) destaca la crisis de los valores y la poca fe y creencia de estos produciendo una desorientación en las personas por la falta de los mismos. Tratan también este tema Spranger, Böhler, Entralgo y Cariacedo (cit. lyanga, 1998). lyanga (1998) y Negrín y Vergara (2003) dan su opinión al comentar el cambio que ha habido en la concepción de los valores tras el paso del tiempo.

En estas teorías se hace referencia a la ilustración y a la modernidad considerándose los ilustrados partícipes de una educación nacional, uniforme, cívica, sin exclusiones asegurando la igualdad para todos los ciudadanos del Estado. Una figura representativa del pensamiento ilustrado es Kant, formado por sus 
principios filosóficos y cívicos. Este filósofo considera que hay que hacer hincapié en modelar el carácter, ser personas independientes y autónomas, forjar nuestra personalidad y conciencia moral afianzando nuestra formación.

Participan de las ideas anteriormente comentadas de la ilustración, resaltando la importancia de los valores cívicos, Mandeville, Paley, Toland, Hume y Clarke (cit. Negrín y Vergara, 2003).

- Teorías de la Postmodernidad y la educación: Nietzche (cit. Colom, 1997 y Negrín y Vergara, 2003) no aboga por los valores es más no los considera necesarios en la vida del ser humano. Negrín y Vergara (2003) sostiene que en la Postmodernidad el ser humano no necesita aferrarse a los valores.

Corrientes críticas de la Escuela. La desescolarización: Illich (cit. Negrín y Vergara, 2003 y Del Pozo, 2004) se centra en los valores de la niñez y en los valores sociales, estando en contra del sistema escolar como mecanismo de adoctrinamiento y jerarquía sin libertad de pensamiento y realización en los educandos.

Del Pozo (2004) considera que la secularización en las escuela públicas suponía una pérdida de los valores cívicos no deseando las familias que sus hijos recibieran educación de esa manera, indoctrinando y enseñando el valor de la competencia, por ello abogaban por un profesorado transformador del conocimiento con una buena dialéctica que contribuyera al conocimiento de la vida real y actual del estudiante, centrado en sus experiencias y en el saber teórico-prácti$\mathrm{co}$, rechazando todo conocimiento impuesto basado en cualquier tipo de ideología dominante del momento. Estas posturas se extienden desde los años 80 y 90 del siglo XX. Mantienen estos pensamientos de igual forma Goodman (cit. Colom, 1997 y Vicente, 2002) y Mcluhan e Illich (cit. Negrín y Vergara, 2003).

- Teorías antiautoritarias: No recogen el tópico valor de forma general sino de forma específica haciendo referencia a valores concretos y determinados.

- Teorías personalistas: Mounier (cit. Negrín y Vergara, 2003 y Del Pozo, 2004) alude a los valores que sean elegidos libre y responsablemente por las personas comprometiéndose estas a llevarlos a cabo en las etapas de sus vidas. Igualmente, Del Pozo (2004) comenta que los personalistas destacan la transmisión de valores basados fundamentalmente en el compromiso.

Scheller y Mounier (cit. Colom, 1997) se refieren a lo cívico en el sentido de acoger al otro, de aceptarlo tal y como es, de fomentar las actitudes y el trabajo en grupo comúnmente.

- Movimiento de la Escuela Nueva: Marín (1988) hace mención a Luzuriaga y García Benítez (2001) a Planchard para comentar que ambos autores destacan la formación de la persona desde el civismo y la cooperación, con un sentido comunitario y colectivo entre todos.

Lietz (cit. Negrín y Vergara (2003) junto con sus colaboradores Wyneken y Geheeb se centran en la coeducación incluyendo en sus programas que los alumnos adquieran y desarrollen caracteres religiosos-cívicos. Comparten estas ideas Rousseau, Pestalozzi, Tolstoi, Key, Froebel y Herbart. Que el ciudadano adquiera preparación realizando juicios de valor en relación a la nación y a la sociedad manteniendo en todo momento una neutralidad confesional. 


\subsection{Resultados: análisis y discusión}

Los diagramas de redes que han sido elaborados con el programa ATLAS.ti se integran dentro de la metodología no sólo como una forma de tomar apuntes o registro, sino como parte de un proceso interpretativo que permite construir relaciones y significados en los discursos representados (García Ponce, Montero y Aguilar, 2004). De acuerdo con Ahmad y Adman (2003), el empleo de representaciones simbólicas, facilita la estructura y el contenido del proceso mental del investigador en convergencia con la realidad estudiada. La disposición de conceptos a través de una representación simbólica favorece la existencia de un pensamiento sistémico que permite adquirir una perspectiva compleja y crítica sobre el discurso. A continuación, se muestra el análisis de los resultados obtenidos con el programa ATLAS.ti.

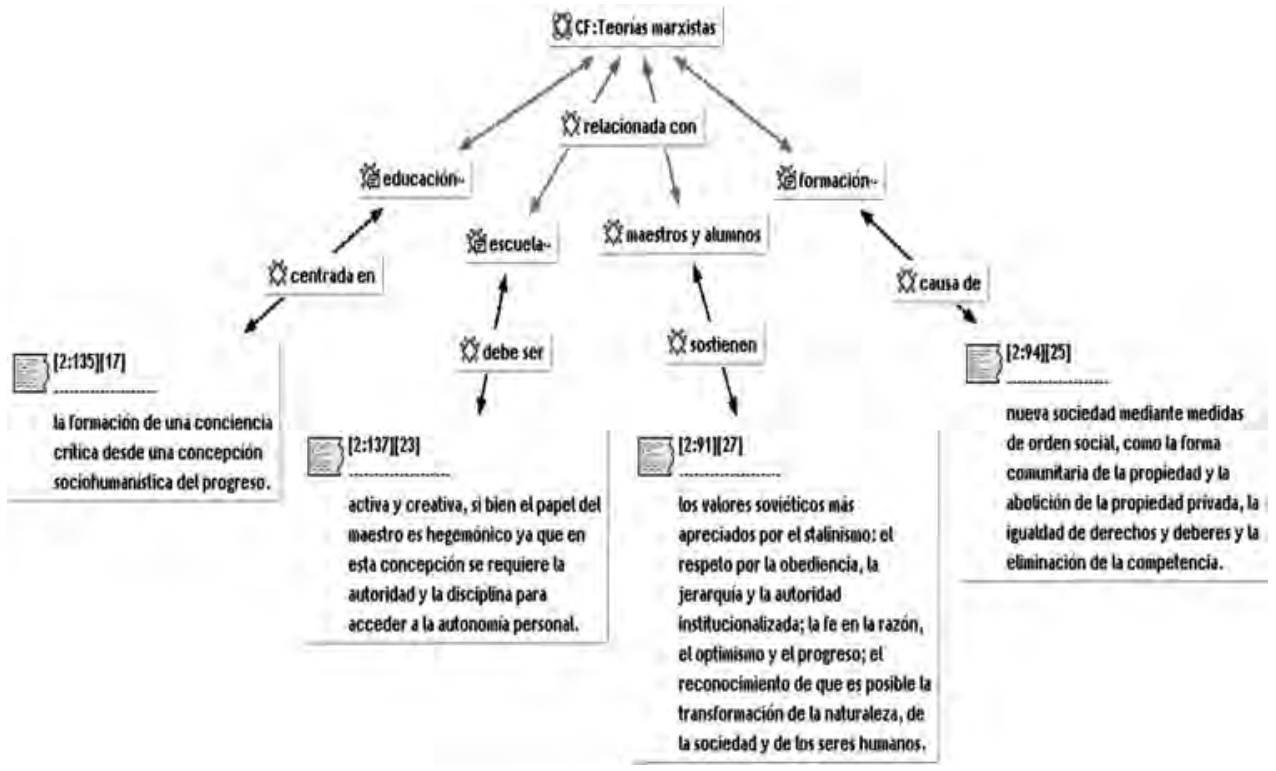

Figura 1. Teorías marxistas

Tal y como se muestra en la figura 1, en las teorías marxistas Bernabeu (1997, 2002, 2008) y Negrín y Vergara $(2003,2006)$ hacen referencia a Suchodolski quien comenta que la educación debe ser contemplada no como mera adaptación para los cambios tecnológicos del progreso y el desarrollo, sino para la realización creadora del trabajo profesional y la participación activa en la vida social, con la consiguiente formación en valores cívicos y el desarrollo de una vida cultural. Carreño (2000, 2002, 2008) y Negrín y Vergara aluden a Makarenko que expone que la disciplina es un aspecto básico en la formación del educando y uno de los más controvertidos de la pedagogía de este educador. Sin embargo, este autor pretendía que no se llevase a cabo sólo una disciplina por la disciplina; por el contrario, habla de "disciplina consciente" que sería aquella que cada persona considera benéfica tanto para la colectividad como para sí. La disciplina está al servicio del colectivo, no es arbi- 
traria sino que surge de las necesidades del grupo y se encamina al buen funcionamiento de éste. Makarenko considera lo colectivo por encima de lo individual. También, Owen dirige su atención no a la formación del individuo, sino a la formación de una nueva sociedad mediante medidas de orden social. Del Pozo (2004, 2009) expone que los maestros y los alumnos interiorizaron los valores soviéticos más apreciados por el estalinismo, entre los cuales no se aprecia la libertad. Aunque en el pensamiento de Marx existe toda una teoría general de la libertad (Prior, 2004).

También esta autora hace referencia a un texto de la Escuela Modelo de Moscú, donde el concepto "cívico" aparece en el Reglamento de la escuela que pedía disciplina consciente, desarrollo político, responsabilidad cívica y conducta cultivada.

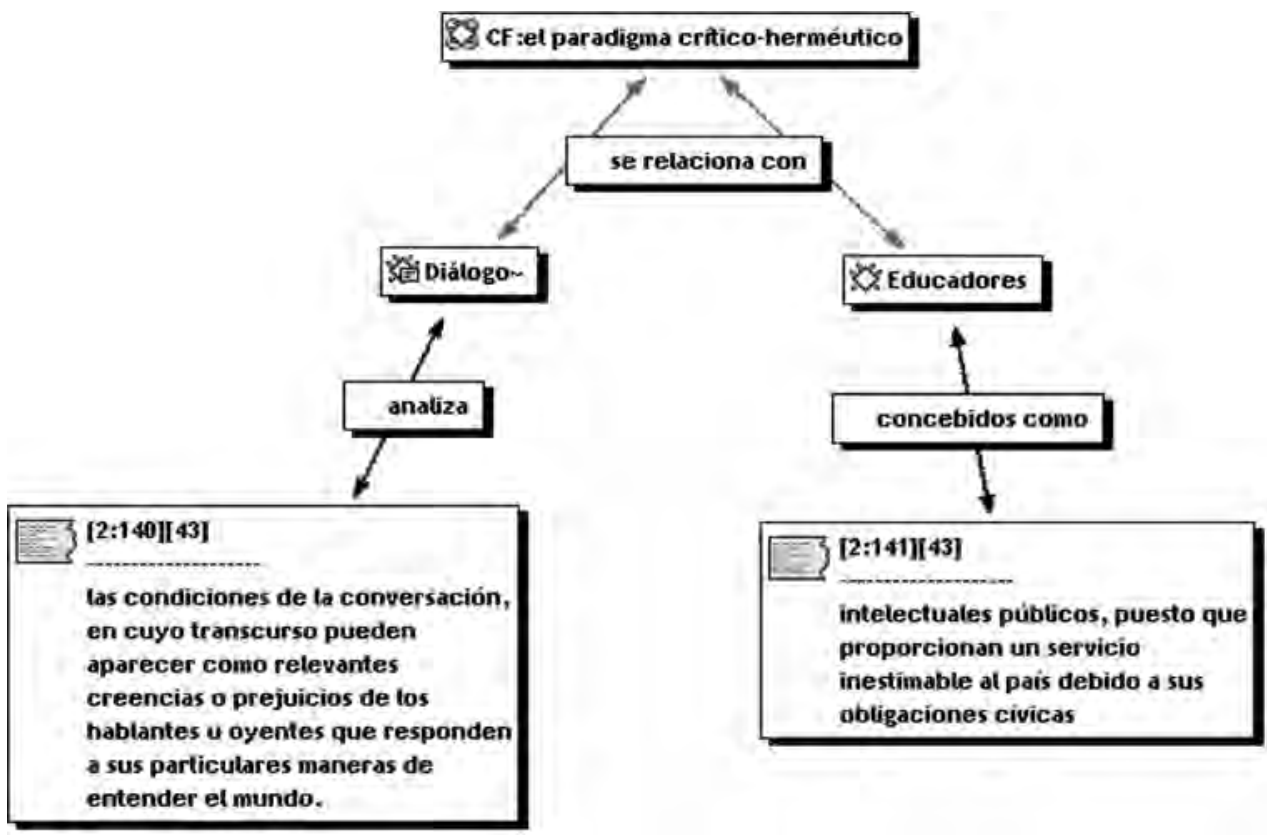

Figura 2. Paradigma crítico-hermenéutico

En el paradigma crítico-hermenéutico, como se recoge en la figura 2, Bernabeu y Colom $(1997,2002,2008)$ y Del Pozo $(2004,2009)$ sostienen que el derecho de los participantes en la conversación a manifestar las opiniones fruto de sus experiencias permite nuevos significados y consensos. La perspectiva comunicacional, basada en las teorías de Habermas, incluye en el diálogo sobre la educación a profesores, estudiantes y los miembros de la sociedad a la que pertenecen. El cambio educativo constituye un desafío para los profesores, dado su papel en la formación de los estudiantes como ciudadanos críticos y activos; sin embargo, el papel que se les asigna corresponde cada vez más con el de ejecutores del aprendizaje, devaluando su trabajo intelectual y crítico. Para salir de la crisis de los profesores, Giroux (1990) reivindica al profesor como intelectual transformativo y comprometido que combine la reflexión con la práctica educativa. Giroux considera a los educadores 
como intelectuales públicos, que proporcionan un servicio inestimable al país debido a sus obligaciones cívicas. Ellos serán los encargados de transmitir las prácticas democráticas en la escuela, en orden a promover la justicia económica y la diversidad cultural por medio de prácticas basadas en el debate, en el diálogo (ética dialógica) y en el intercambio de opiniones. Este nuevo modelo de profesor será el encargado de expandir las prácticas democráticas en la escuela, en orden a promover la justicia económica y la diversidad cultural por medio de prácticas basadas en el debate, en el diálogo y en el intercambio de opiniones.

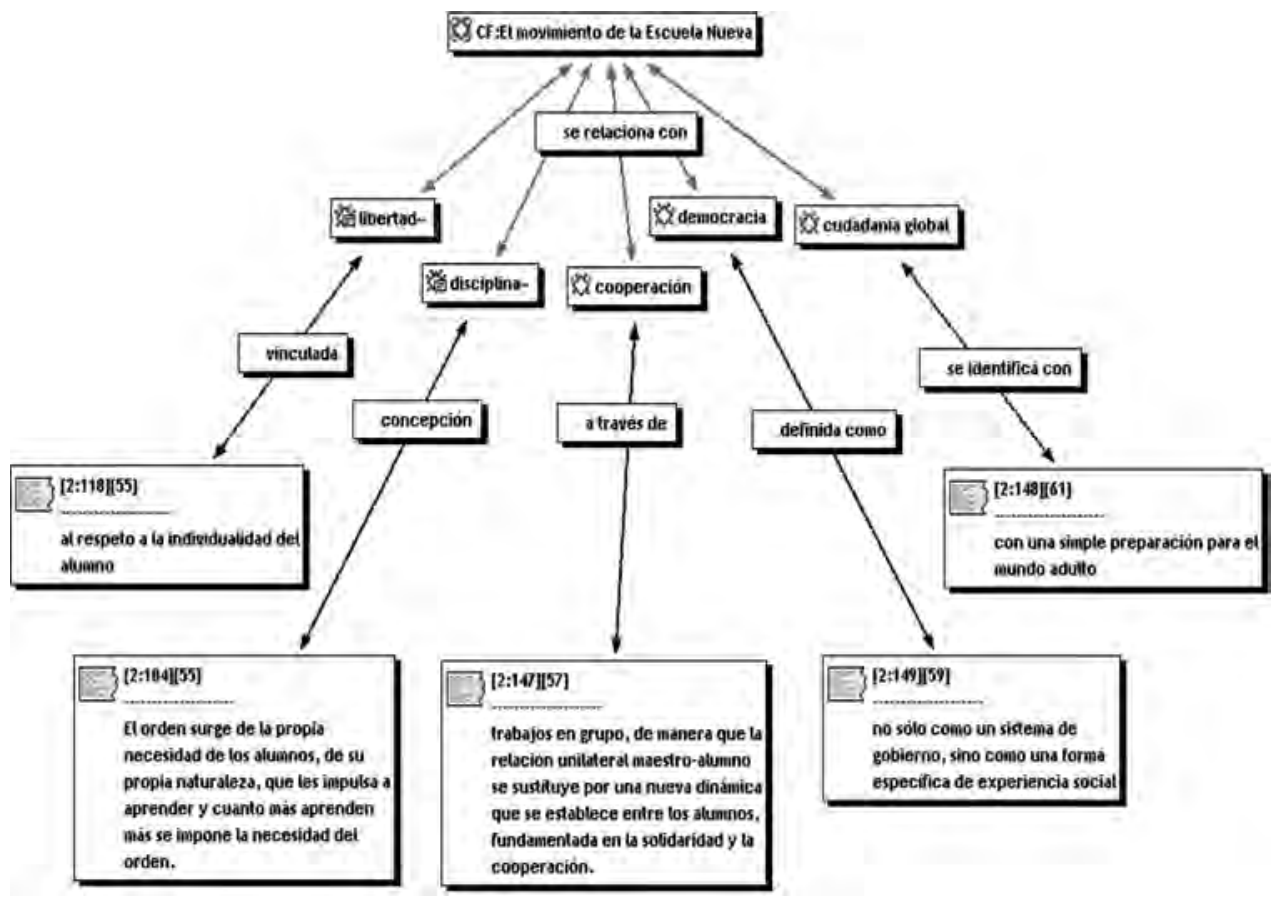

Figura 3. El movimiento de la Escuela Nueva

Tal y como se recoge en la figura 3, el movimiento de la Escuela Nueva, Carreño (2000, 2002, 2008) y Negrín y Vergara $(2003,2006)$ hace referencia a Tolstoi quien comenta que la libertad está estrechamente vinculada al respeto a la individualidad del alumno. En la concepción de Tolstoi de la disciplina el orden surge del desorden, al que llama "orden libre", y lo considera el primer momento de un proceso que culmina en el orden. Si el desorden parece horrible a los maestros es, según este autor, porque han sido instruidos en un sistema autoritario y violento fundado en una mala interpretación de la naturaleza humana. Aunque el desorden parece que aumenta y crece por momentos, solo basta esperar un poco y se extinguirá produciendo un orden más perfecto que el impuesto por el maestro. También, se fomenta la cooperación entre los niños. Se trata de que el individuo no se forme en actividades aisladas, sino mediante trabajos en común con los demás y al servicio de los otros. Eso no significa, sin embargo, el olvido del respeto a la individualidad y a la personalidad infantil que 
conforman aspectos básicos de la Escuela Nueva. Del Pozo (2004, 2009) hace referencia a Dewey quien en su libro Democracia y Educación definía una democracia como un modo de comunicación, como un conjunto de ciudadanos que participan activamente en la construcción y reconstrucción de la sociedad. Para impulsar el ideal democrático de la educación, esto es, para educar ciudadanos demócratas se requería convertir la escuela en una "comunidad embrionaria", sustentada en un espíritu de cooperación y en el desarrollo de una conciencia social y de un pensamiento colectivo de carácter crítico. Para Dewey, la educación ha de ser concebida como una reconstrucción continua de la experiencia. Una educación para todos que constituya la base real de la democracia. Establece un paralelismo entre el método científico y el método educativo, en el que la experiencia es la base de todo aprendizaje. La educación es la posibilidad de aumentar en experiencias y la escuela debe asemejarse y acercarse al máximo al mundo real.

En este sentido, Del Pozo comenta que la finalidad educativa de "preparar para la vida", tan característica de la Escuela Nueva, se empezó a entender como una mirada hacia el futuro con propósitos transformadores, como una forma de reconstruir la sociedad a través de la educación, lo cual implicaba preparar al futuro ciudadano en la comunidad y, aún más, prepararle para una ciudadanía global, para ser parte de la Humanidad.

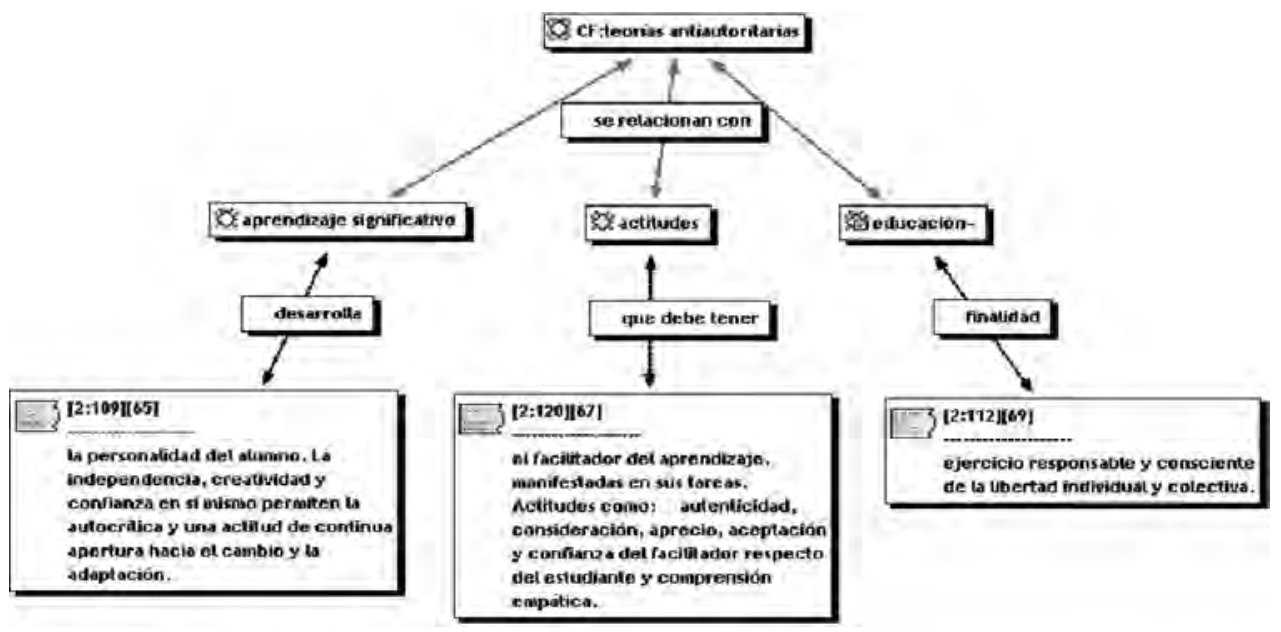

Figura 4. Teorías antiautoritarias

En las teorías antiautoritarias, figura 4, Bernabeu y Colom (1997, 2002, 2008) argumentan que el aprendizaje significativo desarrolla la personalidad del alumno. De igual modo, el alumno con libertad de acción y tiempo, creciendo en su madurez y responsabilidad, se encamina por las sendas del aprendizaje, tanto individual como en grupo, cuya autogestión, tareas y objetivos son asimismo definidos por los estudiantes. Como se muestra, la no directividad también está presente en las relaciones entre profesor y alumnos. Negrín y Vergara $(2003,2006)$ hacen referencia a Rogers y la importancia que este concede a las actitudes que debe tener el facilita- 
dor del aprendizaje, manifestadas en sus tareas. Asimismo, estos autores recogen que el anarquismo reivindica la esencial libertad del individuo y su naturaleza social de tal forma que la educación servirá para que afloren los sentimientos de solidaridad y cooperación. La democracia permanente y directa garantiza formas alternativas de organización en la que es posible desarrollar las potencialidades de los individuos. Del Pozo $(2004,2009)$ relaciona la finalidad de la educación con la libertad individual y colectiva a través del ejercicio responsable y consciente del docente y del educando.

Si hay algo que caracteriza fundamentalmente a este movimiento señala Vicente (2002: 91-110) "es el deseo de educar en libertad y para la libertad". Explica Vicente (2002) que en la escuela convencional, autoritaria y represiva, el grupo pasaba desapercibido, siendo cultivadas únicamente las relaciones verticales entre el profesor y los discípulos, con claro signo de sometimiento a los adultos. Sin embargo, en la pedagogía antiautoritaria y no-directiva, no es el profesor el que monopoliza la escuela; los alumnos usan la palabra dentro de su grupo y cooperan a la educación de sus iguales.

Figura 5. Teoría de la desescolarización

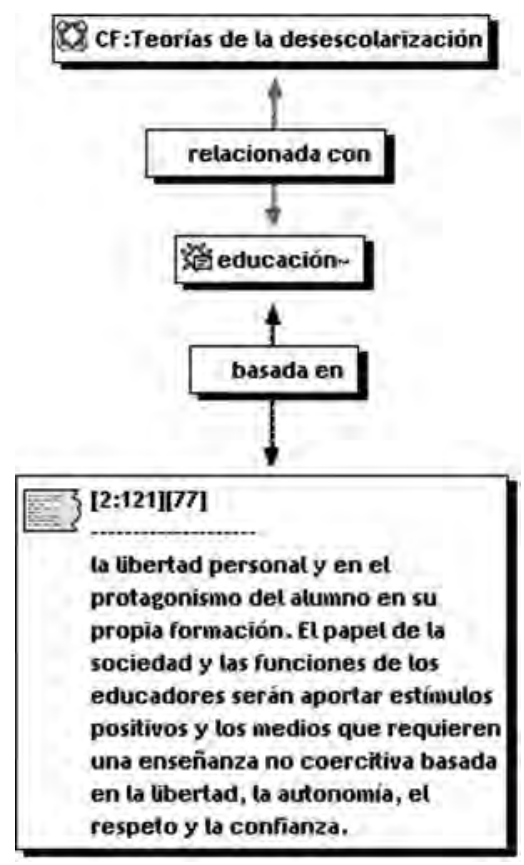

En las teorías de la desescolarización, figura 5, Negrín y Vergara (2003, 2006) hacen referencia a Goodman, su crítica a la escuela existente está relacionada con la capacidad de represión que se ejerce sobre los niños. Ante esta situación represiva, el autor plantea alternativas escolares que permitan una educación no represiva de los alumnos. También analizan a Holt que llevó a cabo de forma práctica la desescolarización preocupándose por la renovación escolar, que implicaba la renovación de la sociedad, una sociedad que deseaba más justa y moral, donde el medio ambiente, la paz, la integración de razas y culturas fuesen una realidad. Para Holt, 
la libertad es responsabilidad. Aconseja a los padres educar a sus hijos desde el hogar a fin de alejarlos y mantenerlos a salvo de las perversiones escolares, haciendo hincapié en la formación del sentimiento, la voluntad, la educación moral y social. Holt consideró que la escuela anulaba las capacidades educativas del alumnado, así como su libertad y espíritu independiente. Con tales planteamientos, e intentando dar la máxima libertad al alumnado, Holt idea un sistema alternativo de formación en el que implica a la familia y pretende suprimir la escuela.

\section{Conclusiones}

El objetivo principal de este trabajo ha sido analizar los valores cívicos en los manuales docentes universitarios de la asignatura "Teorías e Instituciones Contemporáneas de Educación" que se imparte en el Grado de Educación Primaria en las universidades españolas. Para ello se han analizado los valores implícitos en las teorías o corrientes que abordan los manuales de la asignatura seleccionada.

En cada teoría o corriente estudiada se inciden en los siguientes valores: la teoría marxista destaca lo colectivo por encima de lo individual, la escuela basada en los valores de autoridad y disciplina y la formación comunitaria (igualdad de derechos y deberes). El paradigma crítico-hermenéutico establece una relación de diálogo entre el profesor y el alumno porque en el transcurso de la conversación se ponen en juego creencias o prejuicios de los hablantes. El movimiento de la Escuela Nueva alude a la democracia, la ciudadanía, la cooperación, la disciplina y la libertad para preparar al ciudadano en comunidad. Las teorías antiautoritarias se centran en las actitudes porque consideran que facilitan el aprendizaje significativo del alumno, con una educación basada en la libertad individual y colectiva. Las teorías de la desescolarización al igual que la anterior enfatiza la libertad del alumno en su propia formación, fomentando la autonomía, el respeto y la confianza.

En general, ha habido una evolución de los valores a lo largo del tiempo, ya que estos han ido incrementándose y se han notado con más fuerza los conceptos de valores cívicos, ciudadanía y democracia desde el año 2003 hasta la actualidad, sobre todo con la última autora estudiada, Del Pozo (2004, 2009). Asimismo, entre los autores, hay semejanzas de pensamiento en la mayoría porque coinciden en reafirmar los mismos valores o muy parecidos aunque traten distintas teorías. Y entre un mismo autor y sus diferentes ediciones no ha habido apenas diferencias significativas a destacar, aunque ha sido relevante analizar las distintas ediciones a veces en un mismo autor por si variaban los valores en dichos manuales.

Investigar sobre esta asignatura ayuda a mejorar la docencia de la misma y la formación de los maestros teniendo una implicación directa en su formación inicial. La educación cívica despierta bastante interés, tanto en el ámbito de la reflexión como en el de la práctica, pero son escasos los estudios que tratan de analizar la función, el papel, las concepciones y el peso de las mismas en la formación del profesorado. Nuestra idea es ampliar el análisis comparativo con más manuales universitarios de esta misma asignatura para ver la evolución de los valores a lo largo del tiempo. 


\section{REFERENCIAS BIBLIOGRÁFICAS}

ARMAS, X. (2004). Didáctica de las ciencias sociales, geografía e historia, en F. Salvador., J. L. Rodríguez y A. Bolívar (Dirs.), Diccionario Enciclopédico de Didáctica. Málaga: Aljibe, 161-174.

BOLÍVAR, A. (2000). La escuela pública y los valores en el nuevo siglo. Revista de los centros de profesorado de Andalucía, 3, 1-23.

BOLÍVAR, A. (2007). La educación para la ciudadanía algo más que una asignatura. Barcelona: Graó.

BUXARRAIS, M. a R. y MARTÍNEZ, M. (2009). Educación en valores y educación emocional: propuestas para la acción pedagógica. Teoría de la Educación. Educación y Cultura en la sociedad de la información, 10 (9), 320-335.

CAÑAS, A. J.; NOVAK, J. D. y GONZÁLEZ, F. M. (Eds.) (2004). Concept maps: theory, methodology, technology. Pamplona: Dirección de Publicaciones de la Universidad Pública de Navarra, 109-116.

CORTINA, A.; ESCÁMEZ, J. y PÉREZ, E. (1996). Un mundo de valores. Valencia: Generalitat Valenciana.

ESCÁMEZ, J. (2002). La educación de la ciudadanía. Madrid: Editorial CCS.

ESCOLANO, A. (Dir.) (1997-1998). Historia ilustrada del libro escolar en España. Madrid: Fundación Germán Sánchez Ruipérez.

GARCÍA PONCE, O.; MONTERO, V. y AGUILAR, M. F. (2004). Mapas conceptuales aplicados al análisis del discurso de grupos en la universidad, en A. J. Cañas., J. D. Novak y F. M. Giroux, H. A. (1990). Los profesores como intelectuales. Hacia una pedagogía crítica del aprendizaje. Barcelona: Paidós-MEC.

GERVILLA, E. (1992). Los valores y cultura de los jóvenes. Madrid: Dikynson.

GODÁS, A.; SANTOS, M. A. y LORENZO, M. (2009). ¿Qué es lo que importa en educación para la ciudadanía? Evaluación de un cuestionario para profesores. Teoría de la Educación. Revista Interuniversitaria, 21 (2), 95-129.

MACINTYRE, A. (1994). Justicia y racionalidad. Barcelona: Eunsa.

MARÍN, R. (1988). Principios de la educación contemporánea. Madrid: Rialp.

MARTÍN, A. V. y BARRIENTOS, O. (2009). Los dominios del pensamiento crítico: una lectura desde la teoría de la educación. Teoría de la Educación. Revista Interuniversitaria, 21 (2), 19-44.

MELLA, O. (2003). Metodología cualitativa en ciencias sociales y educación: orientaciones teórico-metodológicas y técnicas de investigación. Santiago: Primus.

PAREDES, E. y RIBERA, D. (2006). Educar en valores. Barcelona: Tibidabo.

PÉREZ, J. A. (1996). Claves humanitarias para una educación democrática: de los valores humanos al hombre como valor. Madrid: Anaya.

PÉREZ., G. (1994). Investigación cualitativa. Retos e interrogantes. Madrid: La Muralla.

PRATS, J. (2011). Educación para la ciudadanía en la Universidad. Escuela, 387, 3.

PRIOR, Á. (2004). El problema de la libertad en el pensamiento de Marx. Madrid: Biblioteca Nueva.

PUIG, M.; DOMENE, S. y MORALES, J. A. (2010). Educación para la ciudadanía: referentes europeos. Teoría de la Educación. Revista Interuniversitaria, 22 (2), 85-110. 
PUYOL, E. y LUZ, I. (2003). Valores para la convivencia. Barcelona: Parramón.

ROMÁN, J. M. y CANO, R. (2008). La formación de maestros en España (18382008): necesidades sociales, competencias y planes de estudio. Educación XXI, $11,73-101$.

TORRES, G. (2013). Identidades de género, sexualidad y ciudadanía: un análisis crítico del curriculum de educación sexual integral. Contextos educativos, 16, 41-54.

VALLS, R. (2001). Los nuevos retos de las investigaciones sobre los manuales escolares de Historia. Revista de Teoría y Didáctica de las Ciencias Sociales, 6, 31-42.

VILLALAÍN, J. L. (1997). Manuales escolares en España. Madrid: UNED. 